\title{
Time series analysis with apache spark and its applications to energy informatics
}

\author{
Cornelia Krome* and Volker Sander \\ From The 7th DACH+ Conference on Energy Informatics \\ Oldenburg, Germany. 11-12 October 2018
}

\author{
* Correspondence: krome@fh- \\ aachen.de \\ Faculty of Medical Engineering and \\ Technomathematics, \\ Fachhochschule Aachen, 52428 \\ Jülich, Germany
}

\begin{abstract}
In energy economy forecasts of different time series are rudimentary. In this study, a prediction for the German day-ahead spot market is created with Apache Spark and R. It is just an example for many different applications in virtual power plant environments. Other examples of use as intraday price processes, load processes of machines or electric vehicles, real time energy loads of photovoltaic systems and many more time series need to be analysed and predicted.

This work gives a short introduction into the project where this study is settled. It describes the time series methods that are used in energy industry for forecasts shortly. As programming technique Apache Spark, which is a strong cluster computing technology, is utilised. Today, single time series can be predicted. The focus of this work is on developing a method to parallel forecasting, to process multiple time series simultaneously with $R$ and Apache spark.
\end{abstract}

Keywords: Time series, Apache spark, Energy informatics, Forecast, ARMA

\section{Content}

At EPEX Spot exchange electricity is traded and exchange members can offer bids for buying and selling energy (EPEX Spot SE, 2018). In Germany bids for the next day must be submitted until noon. Normally, single hours are traded at the day-ahead market. The Market Clearing Price reveals as intersection between offered and demanded amount for each hour. In the end, every market member pays this price for a particular hour (next-Kraftwerke, 2018). The price process for January to June 2018 can be seen in Fig. 1.

In context of the EFRE.NRW funded project KundenoRientiert FlexibilisierungspoTenziale erschließen (KRaFT) we are interested in price and load profile forecasting. Today, most platforms focus on a single time series. When applying the forecast of time series to more sources, we are facing big data problems. One example of use may be the analysis of load profiles for each electric vehicle load station in a car park. So, strategies for best loading can be applied and may

(c) The Author(s). 2018 Open Access This article is distributed under the terms of the Creative Commons Attribution 4.0 International License (http://creativecommons.org/licenses/by/4.0/), which permits unrestricted use, distribution, and reproduction in any medium, provided you give appropriate credit to the original author(s) and the source, provide a link to the Creative Commons license, and indicate if changes were made. 


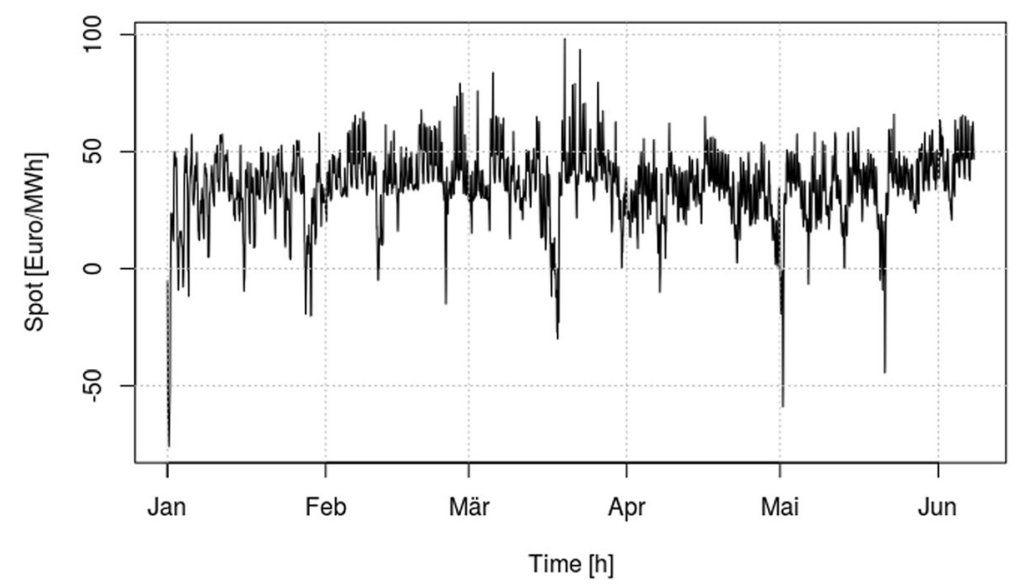

Fig. 1 Time series of German day-ahead prices in 2018

maximise profit. At the moment, the given time series can be analysed and predicted with $R$ ( $\mathrm{R}$ Core Team, 2018). But for new use cases $R$ itself is not sufficient.

In this work it is analysed, how multiple time series can be processed. Therefore, parallel computing with $R$ and the clustering tool Apache Spark (Apache, 2018a) is used. We analyse the feasibility of computing those to make differentiated forecasts for several datasets in parallel, allowing complex forecast scenarios in a virtual power plant environment as it is addressed by the KRaFT project.

\section{Time series method}

This section provides a short summary of the autoregressive-moving average (ARMA) models, which are used to predict day-ahead prices.

ARMA-models are a mix of autoregressive (AR) and moving average (MA) models. AR-models relate the current value $\tilde{x}$ of a process to a finite, linear combination of previous values of the process and a random noise $\omega$. They get abbreviated as $A R(p)$, where $p$ describes the order. Instead, MA-models represent $\tilde{x}$ linearly dependent on a finite number $q$ of previous random noise $\omega$ 's (Box et al., 2008).

With a mix of both model types more flexibility is achieved. This leads to ARMA models of order $p$ and $q$, ARMA(p, q) (Box et al., 2008).

$$
\tilde{x}_{t}=\underbrace{\sum_{i=1}^{p} \phi_{i} \tilde{x}_{t-i}}_{\text {AR part }}+\underbrace{\sum_{j=1}^{q} \theta_{j} \omega_{t-j}}_{\text {MA part }}
$$

where $\phi_{i}$ and $\theta_{j}$ are the model coefficients.

\section{Apache spark}

With Apache Spark fast and general-purpose cluster computing can be performed. It can run computations in memory and is recommended for a usage 
with big data which are analysed parallel. The three main components of Apache Spark are Spark Core with the basic functionality, Spark SQL for working with structured data and Spark Streaming to process live streams of data (Karau, 2015) such as day-ahead prices from EPEX Spot or real-time energy loads of photovoltaic systems and electric vehicle load stations.

At the moment, the used architecture saves daily processed day-ahead prices from ENTSO-E Transparency Platform (ENTSO-E Transparency Platform, 2018) in a Hadoop Distributed File System (HDFS) on a cluster where Spark runs standalone. Within each time series analysis, the data is imported as HDFS-file and parallel predicted with Spark. The output can be stored as HDFS-files or with databases as Apache Cassandra (Apache, 2018b), which provides better perspectives for the distribution of multiple time series among the cluster.

In the following sections we analyse an energy price time series with $R$ and execute it on a Spark-Cluster in parallel. To use Apache Spark a session is needed. In $R$ it is started with the following commands:

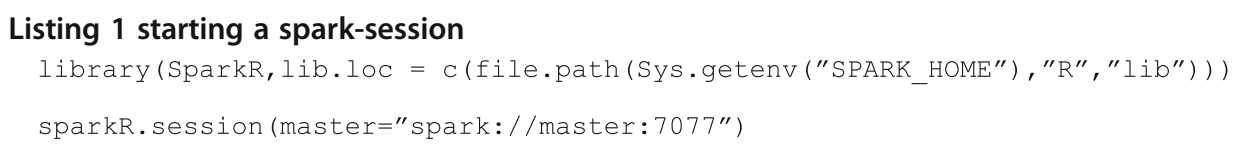

\section{Time series analysis of energy prices}

The data for this analysis are taken from (ENTSO-E Transparency Platform, 2018). A script stores the day-ahead prices on the cluster. The time series of the price process can be seen in Fig. 1.

To compute a forecast, a Spark-session is started first. Subsequently, the day-ahead prices of the German energy market get imported as HDFS-file. Then the time series is created. Here the values for 2018 until June 7th are used. The dataset is divided into training and test data (the last day), which gets predicted.

There is no time series package for Apache Spark and $R$ (R Core Team, 2018) on the market. So, another method to perform forecasts with $R$ on a Spark-Cluster is needed. With the API SparkR the function spark.lapply () (Apache, 2018c) is introduced. Similar to known $R$-functions as apply, sapply and lapply, it runs a user defined function over a list of elements. For each element the $\mathrm{R}$ driver sends the function to an $\mathrm{R}$ worker, executes it and returns the result of all workers as a list to the $\mathrm{R}$ driver. With this possibility a forecast logic is written in $R$ and is parallel performed for multiple time series on a Spark-Cluster. The command for running this is shown in Listing 2.

Listing 2 command for spark.Lapply fcast <- spark. lapply (liste, getForecast)

Multiple time series, which are available as distributed datasets in the cluster, are included in liste. The user defined function getForecast (see Listing 3) processes the prediction for each element in liste. 
Listing 3 method used in lapply

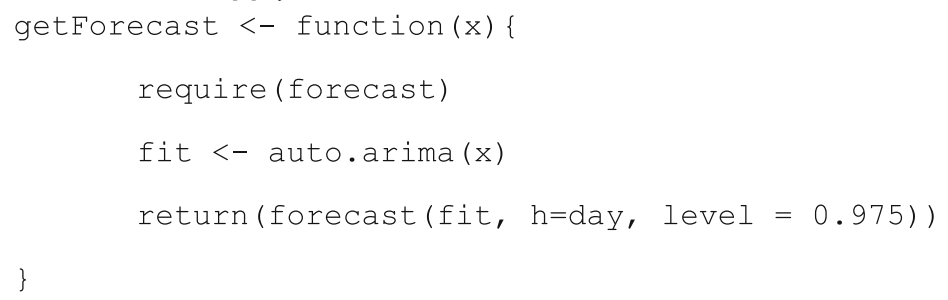

All required packages need to be loaded within the function. The model of the time series is created with auto.arima, a known $R$-function (Hyndma \& Khandakar, 2008). In this case, the time series of the day-ahead prices is modelled with an ARMA $(3,2)$ with non-zero mean model. With the $R$-function forecast (Hyndma \& Khandakar, 2008) a prediction of the next 24 values (1 day) is calculated based on the training process.

In Fig. 2 the forecast (red) is shown. Additionally, the historic values of 1 week (black), the real observations for the last day (green) and the confidence interval for the prediction (blue) is plotted.

\section{Summary}

To submit bids for electricity at the EPEX Spot a good knowledge of needs and a price forecast is recommended. Therefore, different methods can be used, for instance a simple ARMA-model can predict the first few hours of a day. Other models and a preceded clustering of the data may bring a better result.

The first test of Apache Spark and $R$ showed, that even if there is no special package for time series analysis in $R$ with Apache Spark, it is possible to run parallel forecasts on a Spark-Cluster in $R$. So, multiple time series of price processes or load profiles like those of electric vehicle load stations in a car park, can be done simultaneously.

In future works, the scalability of time series analyses with Apache Spark and $R$ with the developed method will be thematised.

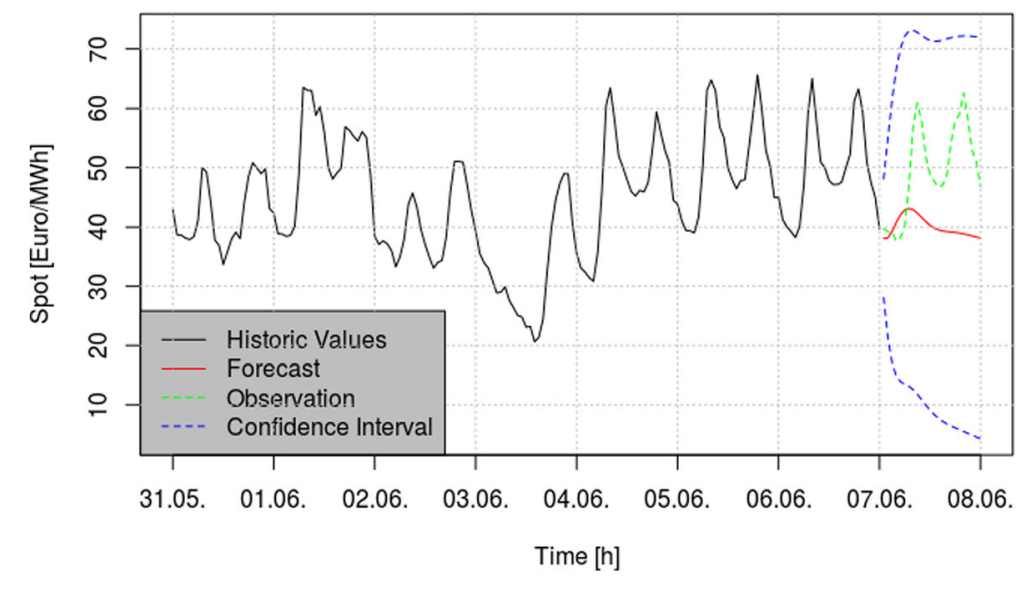

Fig. 2 Forecast of day-ahead prices of June 7th 2018 


\section{Abbreviations}

AR: Autoregressive; ARMA: Autoregressive-moving average; HDFS: Hadoop Distributed File System;

KRaFT: KundenoRientiert FlexibilisierungspoTenziale erschließen; MA: Moving average

\section{Funding}

Publication of this article was sponsored by EFRE.NRW grant.

Publication costs for this article were sponsored by the Smart Energy Showcases - Digital Agenda for the Energy Transition (SINTEG) programme.

\section{Availability of data and materials}

Datasets related to this article can be found at https://transparency.entsoe.eu/transmission-domain/r2/dayAheadPrices/ show, with area Germany, BZN|DE-AT-LU and year 2018 (ENTSO-E Transparency Platform, 2018).

\section{About this supplement}

This article has been published as part of Energy Informatics Volume 1 Supplement 1, 2018: Proceedings of the 7th DACH+ Conference on Energy Informatics. The full contents of the supplement are available online at https://energyinformatics.spr ingeropen.com/articles/supplements/volume-1-supplement-1.

\section{Authors' contributions}

Both CK and VS contributed to the final version of the manuscript. Both authors read and approved the final manuscript

\section{Competing interests}

The authors declare that they have competing interests as they are working on a project called KundenoRientiert FlexibilisierungspoTenziale erschließen which is sponsored by EFRE.NRW.

\section{Publisher's Note}

Springer Nature remains neutral with regard to jurisdictional claims in published maps and institutional affiliations.

Published: 10 October 2018

\section{References}

Apache. Apache Spark - Unified Analytics Engine for Big Data. [Online] 17. 07 2018a. https://spark.apache.org/

Apache. Apache Cassandra. [Online] 17. 07 2018b. http://cassandra.apache.org/

Apache. R: Run a function over a list of elements, distributing the... [Online] 07.06 2018c. https://spark.apache.org/docs/2.0.2/ api/R/spark.lapply.html

Box GE, Jenkins P, Gwilym M, Reinsel GC (2008) Time Series Analysis - Forecasting and Control, vol 4. John Wiley \& Sons, Inc., New Jersey

ENTSO-E Transparency Platform. ENTSO-E Transparency Platform. [Online] 19. 06 2018. https://transparency.entsoe.eu/ EPEX Spot SE. About EPEX SPOT. [Online] 19.06 2018. http://www.epexspot.com/en/company-info/about_epex_spot Hyndma RJ, Khandakar Y (2008) Automatic time series forecasting: the forecast package for $\{R\}$. J Stat Softw 26:1-22 Karau $\mathrm{H}$ et al (2015) Learning Spark: lightning-fast data analysis. O’Reilly, Beijing

next-Kraftwerke. Day-Ahead-Handel - Was Ist Das? [Online] 19. 06 2018. https://www.next-kraftwerke.de/wissen/strommarkt/ day-ahead-handel

R Core Team. R: a language and environment for statistical computing. [Online] 2018. https://CRAN.R-project.org/package=sparklyr

\section{Submit your manuscript to a SpringerOpen ${ }^{\circ}$ journal and benefit from:}

- Convenient online submission

- Rigorous peer review

- Open access: articles freely available online

- High visibility within the field

- Retaining the copyright to your article

Submit your next manuscript at $\boldsymbol{\nabla}$ springeropen.com 